\title{
Acupuncture for Shoulder Pain After Stroke: A Systematic Review
}

\author{
Jung Ah Lee, PhD, Si-Woon Park, MD, MSCR, 2,3 Pil Woo Hwang, MB, Sung Min Lim, MD, \\ Sejeong Kook, $\mathrm{PhD},{ }^{4}$ Kyung In Choi, $\mathrm{MD}^{2}$, and Kyoung Sook Kang, $\mathrm{MD}^{1,5}$
}

\begin{abstract}
Objectives: Shoulder pain, for which acupuncture has been used, is a common complication after a stroke that interferes with the function of the upper extremities. The aim of this systematic review is to summarize and evaluate the effects of acupuncture for shoulder pain after stroke.

Methods: Randomized controlled trials (RCTs) involving the effects of acupuncture for shoulder pain, published between January 1990 and August 2009, were obtained from the National Libraries of Medicine, MEDLINE ${ }^{\circledR}$, CINAHL, AMED, Embase, Cochrane Controlled Trials Register 2009, Korean Medical Database (Korea Institute of Science Technology Information, DBPIA, KoreaMed, and Research Information Service System), and the Chinese Database (China Academic Journal).

Results: Among the 453 studies that were obtained (300 written in English, 137 in Chinese, and 16 in Korean), 7 studies met the inclusion criteria for this review. All of them were RCTs published in China and reported positive effects of the treatment. The quality of the studies was assessed by the Modified Jadad Scores (MJS) and the Cochrane Back Review Group Criteria List for Methodologic Quality Assessment of RCTs (CBRG); the studies scored between 2 and 3 points on MJS, and between 4 and 7 points on CBRG.

Conclusions: It is concluded from this systematic review that acupuncture combined with exercise is effective for shoulder pain after stroke. It is recommended that future trials be carefully conducted on this topic.
\end{abstract}

\section{Introduction}

$S^{3}$ HOULDER PAIN IS A COMMON complication after stroke, interfering with the function of the upper extremities, regular daily activities, and active rehabilitation. ${ }^{1,2}$ The prevalence of shoulder pain relative to the degree of paralysis ranges from $16 \%$ to $84 \% .^{3}$ Over $70 \%$ of stroke patients experience significant shoulder pain. ${ }^{4}$

Shoulder pain after stroke has been connected with contracture of the shoulder, glenohumeral subluxation, injury of the axillary nerve, rupture of the rotator cuff, and spasticity of shoulder muscles, and it is associated with poor motor recovery. ${ }^{2}$ Therefore, it is very important for stroke patients to treat shoulder pain properly. For good motor recovery, various treatments have been suggested, including electrical stimulation, ultrasound, acupuncture, herbal medicine, exercise, taping, and physical therapy.

Acupuncture has been practiced for thousands of years, and it has been widely used in various conditions that in- clude pain, musculoskeletal disorders, and several neurologic disorders. ${ }^{5,6}$ Recent articles reviewing the efficacy and safety of acupuncture in stroke have appeared, but no clear effects of acupuncture were shown. ${ }^{7,9}$ In the literature, there has been no systematic review assessing the effects of acupuncture for shoulder pain following stroke. Therefore, the aim of this study was to summarize and evaluate the results of relevant trials involving this area.

\section{Methods}

\section{Search methods for identification of relevant studies}

Randomized controlled trials (RCTs) reporting effects of acupuncture for shoulder pain, published between January 1990 and August 2009, were obtained from the National Libraries of Medicine, MEDLINE ${ }^{\circledR}$, CINAHL, AMED, Embase, the Cochrane Controlled Trials Register 2009, Korean Medical database (Korea Institute of Science Technology

\footnotetext{
${ }^{1}$ Department of Motor \& Cognitive Rehabilitation, Korea National Rehabilitation Research Institute, Seoul, Republic of Korea.

${ }^{2}$ Department of Rehabilitation Medicine, Korea National Rehabilitation Center, Seoul, Republic of Korea.

${ }^{3}$ Myongji Choonhey Rehabilitation Hospital, Seoul, Republic of Korea.

${ }^{4}$ Department of Statistics, Duksung Women's University, Seoul, Republic of Korea.

${ }^{5}$ Jangdeuk Oriental Medicine Clinic, Seoul, Republic of Korea.
} 
Information, DBPIA, KoreaMed, and Research Information Service System), and the Chinese database (China Academic Journal). Articles written in the English, Korean, and Chinese languages were included in the search. The search string used was (stroke OR cva OR cerebrovascular accident OR intracranial hemorrhage OR ich OR cerebral infarction) AND (shoulder pain OR adhesive capsulitis OR subluxation OR RSD OR CRPS) AND (acupuncture OR electroacupuncture OR electrical stimulation).

\section{Criteria for considering studies for this review}

All prospective randomized controlled clinical studies of acupuncture for shoulder pain after stroke were considered. Trials that included patients of any age or sex, with any type of stroke, and with or without computed tomography scan were eligible. Trials were included if they were randomized studies of acupuncture or electroacupuncture as the sole treatment or as combined with other treatments. Trials that compared two different types of acupuncture were excluded.

\section{Quality assessments for this review}

Two (2) independent reviewers screened titles and abstracts of the database and searched for relevant articles according to predefined criteria. These reviewers also assessed the quality using the Modified Jadad Score (MJS) ${ }^{10,11}$ and the Cochrane Back Review Group Criteria List for Methodologic Quality Assessment of RCTs (CBRG). ${ }^{12}$

The MJS included the following criteria: method of randomization, double blinding, and reporting of withdrawal and dropouts. The CBRG was also examined, including the randomization method, concealment of treatment allocation, similar baseline, assessor blinding, selection of co-intervention, treatment compliance, dropouts, outcome measurement method, and intention-to-treat analysis. Quality assessment was performed by 2 independent reviewers, and disagreements were resolved by discussions between them.

\section{Results}

\section{Study description}

Among the 453 studies obtained from the authors' search (300 written in English, 137 in Chinese, and 16 in Korean),

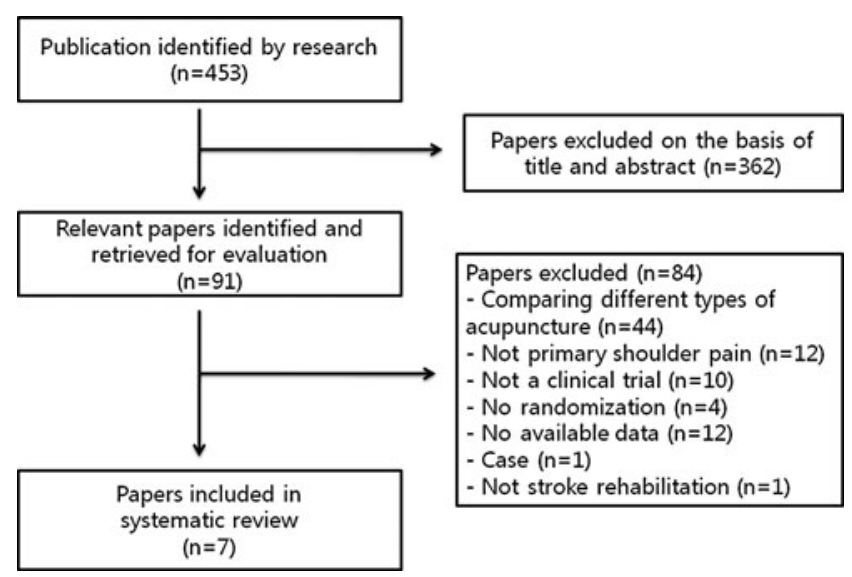

FIG. 1. Flowchart of the trial selection of studies included in the review.
362 studies were excluded on the basis of title and abstract outright (Fig. 1). Then an additional 84 studies were excluded as comparing different types of acupuncture, not relevant to primary shoulder pain, not a clinical trial, not a randomized trial, data not available, no more than a case report, or not relevant to stroke rehabilitation. Seven (7) studies finally met the inclusion criteria. All of them were RCTs published in China, and the results they describe all showed positive acupuncture effects.

Among the seven included studies, four compared a combined acupuncture/exercise treatment with exercise alone, one compared a combined acupuncture/exercise with drug treatment, one compared a combined acupuncture/ exercise with combined drug treatment/exercise, and one compared electroacupuncture with transcutaneous electrical nerve stimulation (TENS) treatment (Table 1).

\section{Study quality}

The average assessment of quality based on the MJS of each study was moderate (mean of 2.1, range of 2-3) (Table 1 ). Among the seven studies, six studies were scored with 2 points ( 1 point for the methods of randomization, and 1 for withdrawals/dropouts). One (1) study was scored with 3 points ( 1 point for the methods of randomization, 1 for blinding, and 1 for withdrawals/dropouts).

The average of quality using the CBRG score was moderate (mean of 5.4, range of 4-7) (Fig. 2). The CBRG was presented in Figure 1. According to this measure, the studies were insufficient in adequate randomization, concealed allocation, patient blinding, assessor blinding, and similar outcome assessment.

\section{Outcomes}

The study of Shang ${ }^{13}$ was divided into three groups: acupuncture combined with exercise, acupuncture alone, and exercise alone. There were significant differences between the combined acupuncture/exercise and the acupuncture-alone groups in outcome determined by the Fugl-Meyer Assessment (FMA), visual analogue scale (VAS), and range of motion (ROM). There was also a significant difference between the combined acupuncture/exercise and exercise-alone groups in outcome determined by the same tests.

Among the seven studies included in this review, three studies compared combined acupuncture/exercise to exercise alone for treating shoulder pain. Three (3) studies found that there was significant improvement in all outcomes determined by $\mathrm{VAS}^{14,16}$, $\mathrm{ROM}^{14}$, clinical score of neural lesion $^{14}, \mathrm{FMA}^{15}$, and Brunnstrom's classification of upper $\operatorname{limbs}^{16}$ after intervention.

Xiong ${ }^{17}$ suggested that the outcome measure of ROM was significantly superior in the group treated with acupuncture combined with rehabilitative exercises rather than drug treatment.

Zhang $^{18}$ found that there were significant differences in outcome measured by VAS and FMA in the combined acupuncture/exercise group compared to combined drug treatment/exercise. However, the outcome measure by Modified Ashworth Scale, Manual Muscle Test, Activity of Daily Living, and Brunnstrom's classification of upper limbs indicated no differences between the two groups. 


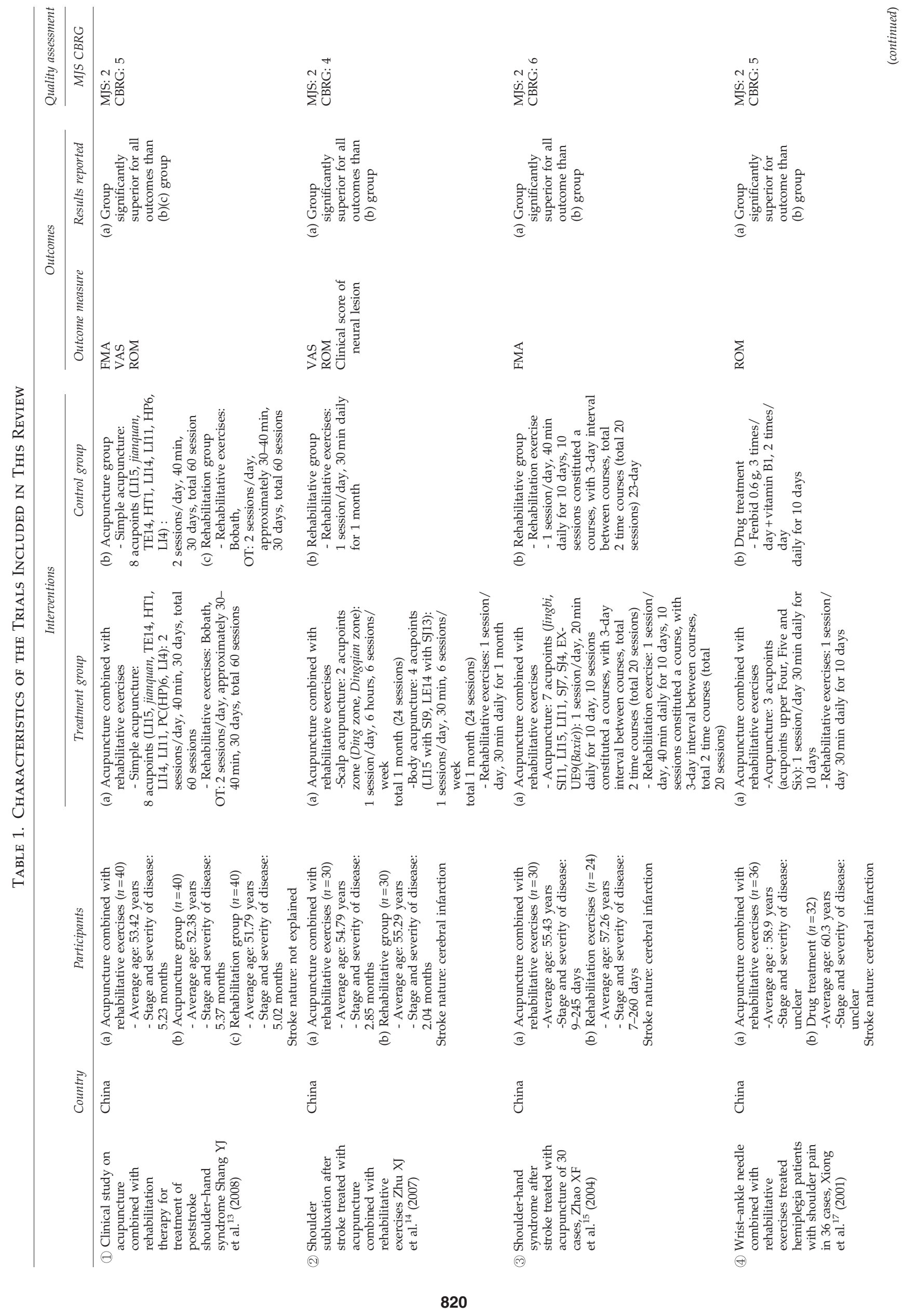




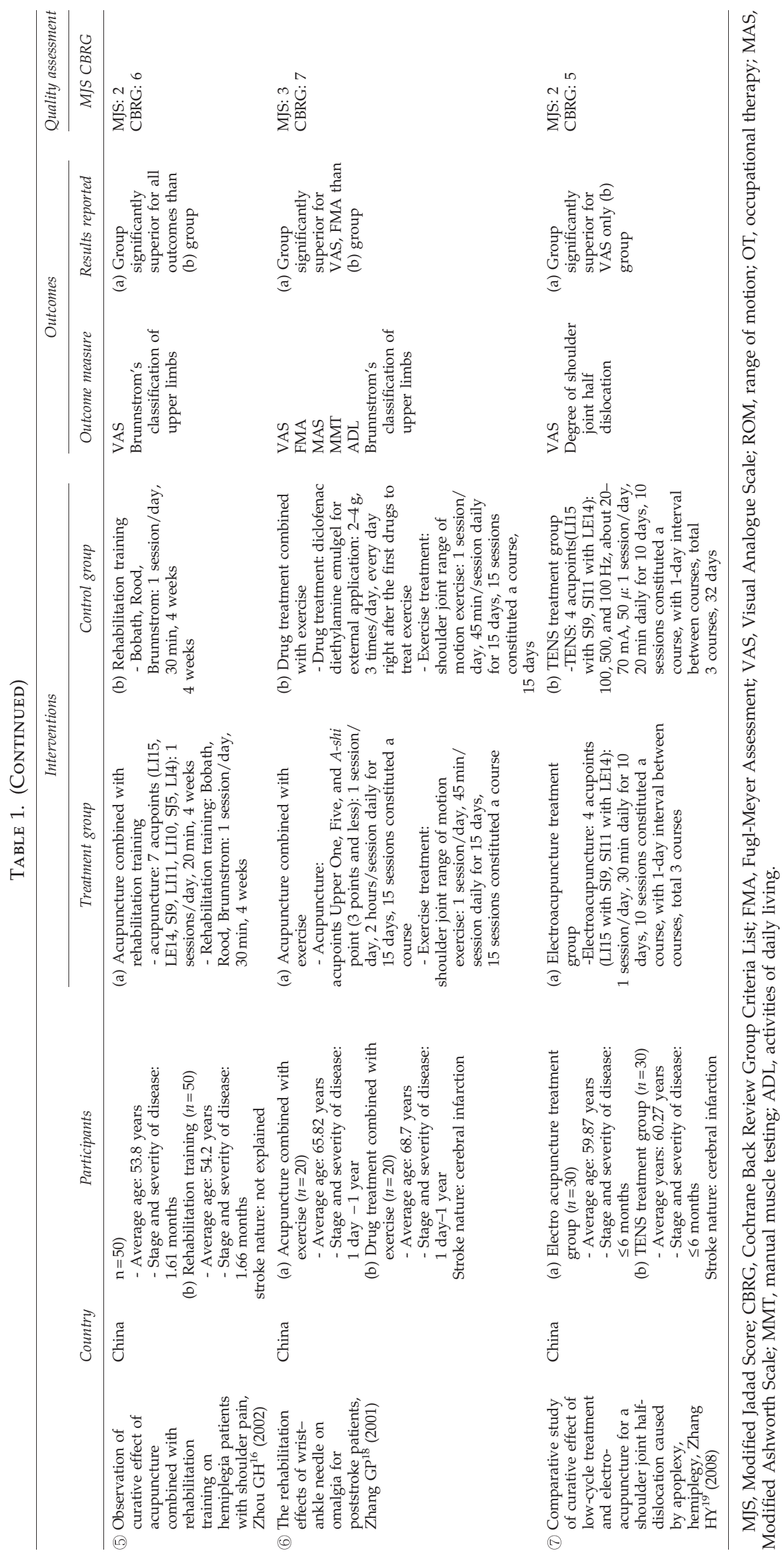


FIG. 2. Assessment of the quality of the studies included in the review.

\begin{tabular}{|c|c|c|c|c|c|c|c|c|c|c|c|}
\hline & 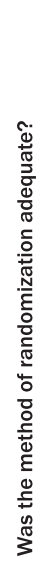 & 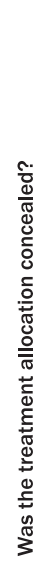 & 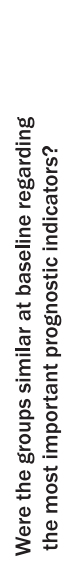 & 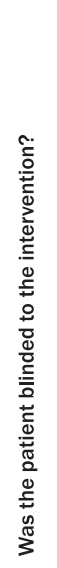 & 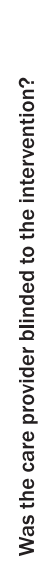 & 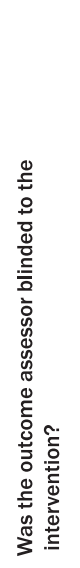 & 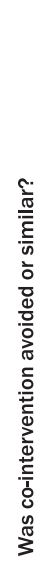 & 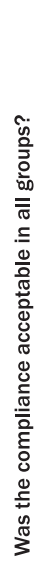 & 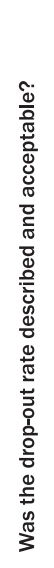 & 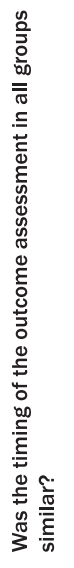 & 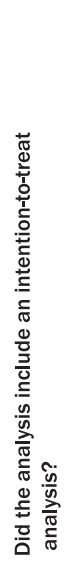 \\
\hline Shang. YJ 2008 & $?$ & $?$ & + & $?$ & $?$ & $?$ & $?$ & + & + & ? & + \\
\hline Zhu. XJ 2007 & $?$ & $?$ & + & $?$ & $?$ & $?$ & $?$ & + & + & $?$ & + \\
\hline Zhao. XF 2004 & $?$ & $?$ & + & $?$ & $?$ & $?$ & + & + & + & + & + \\
\hline Xiong. XR 2001 & $?$ & $?$ & $?$ & $?$ & $?$ & $?$ & $?$ & + & + & + & + \\
\hline Zhou. GH 2002 & $?$ & $?$ & + & $?$ & $?$ & $?$ & + & + & + & + & + \\
\hline Zhang. GP 2001 & $?$ & $?$ & + & $?$ & $?$ & + & + & + & + & + & + \\
\hline Zhang. HY 2008 & $?$ & $?$ & + & $?$ & $?$ & $?$ & + & + & + & ? & + \\
\hline
\end{tabular}

Zhang $^{19}$ showed that the outcome measure of pain by VAS was significantly lower in the electroacupuncture group than in the TENS group, whereas the outcome measure in degree of shoulder joint half dislocation indicated no difference between the two groups.

For the studies using the same measurements, three studies examining acupuncture effect on shoulder pain after stroke recorded changes in VAS after intervention. According to those studies, there was a significant effect in relieving the shoulder pain after acupuncture combined with exercise, which was more effective compared against the exercise alone. Shang, ${ }^{13} \mathrm{Zhu}^{14}$ and $\mathrm{Zhou}^{16}$ used a pain-intensity scale by VAS that ranged between 0 and 5, 0 and 75, and 0 and 100, respectively. Even though these studies had not used standardized pain scale as an outcome measure, three studies reported that acupuncture was effective in improving the shoulder pain.

\section{Discussion}

This systematic review provides suggestive evidence for the effectiveness of acupuncture in treating shoulder pain after stroke. However, the total numbers of RCTs were too small to draw concrete conclusions about the effectiveness of the therapeutic approach. Several other systematic reviews assessing the effects of acupuncture for stroke have been published. ${ }^{20,21}$ Most of them focused on the recovery of general status after stroke in rehabilitation, as measured by the Barthel Index, Nottingham Health Profile, or Scandinavian Stroke Scale. There were several limitations in the evidence showing an effect of acupuncture for stroke.

Zhang et al. ${ }^{8}$ reported that acupuncture appeared to be safe but without clear evidence of benefit in cases of acute stroke. Also, $\mathrm{Wu}$ et al. ${ }^{7}$ concluded that there is no clear evi- dence relating to the effects of acupuncture in subacute or chronic stroke.

The systematic review performed for this report study focused on uncovering evidence of the effects of acupuncture for shoulder pain after stroke, instead of a broader assessment of pain after stroke. Therefore, all relevant trials relating to the effects of acupuncture on shoulder pain after stroke were identified, including studies reported in both Western and Eastern databases. Among the 453 studies retrieved, only 7 of them met the inclusion criteria for this article. All seven studies were RCTs performed in China, and six showed favorable results for combined acupuncture/exercise compared with a control group, such as acupuncture or exercise alone, or a combined drug treatment/exercise or drug treatment regimen. One (1) study showed favorable results for electroacupuncture compared with TENS treatment. For measurement of outcome, VAS was used as the pain scale in the five studies. This scale is a common tool for evaluating pain intensity. Pain relief is an important treatment in stroke rehabilitation or common rehabilitation. In the quality assessment, by MJS the studies scored in the range of $2-3$, and by CBRG in the range of 4-7. Even though the average assessments of quality were moderate, the systematic review provides appropriate significant evidence showing the effectiveness of acupuncture in treating shoulder pain after stroke.

"Acupuncture" means "puncture with a needle" (acus in Latin means "needle"). Acupuncture has been found to relieve pain, sometimes quite dramatically, in many chronic conditions. $^{22}$ There is no clear evidence of the effects of acupuncture on stroke rehabilitation. Acupuncture has biologic effects that might improve recovery from stroke or facilitate rehabilitation. ${ }^{7}$ Properly performed acupuncture seems to be a safe procedure. Basic scientific research is 
beginning to provide evidence that offers plausible mechanisms for the presumed physiologic effects of acupuncture. Multiple research approaches have shown that acupuncture activates endogenous opioid mechanisms. ${ }^{23}$

Acupuncture is a nondrug therapy that is being used more and more often to treat shoulder pain. It is thought that acupuncture works by releasing chemical compounds in the body that relieve pain, by overriding pain signals in the nerves, or by allowing energy (qi) or blood to flow freely through the body. ${ }^{2}$ Although the exact mechanism of this treatment remains unclear, it is apparent that reproducible neurological and chemical changes occur in response to acupuncture, and that these changes almost certainly modify the response to perception of pain. ${ }^{24}$

Even though this review has provided many positive conclusions, there are also several limitations. Although great efforts were made to retrieve all trials on the subject, only 7 studies among 453 studies that were retrieved met the inclusion criteria. All of them were published in China, and all showed positive results related to the effects of acupuncture. Moreover, it was not possible to perform a funnel plot for checking publication bias in this systematic review because of the limited number of trials for each outcome.

In addition, there were insufficient quality assessments with respect to allocation concealment, blinding of outcome assessors, and long-term follow-up. Therefore, larger methodologically sound trials would be required in order to confirm or refute the available evidence.

\section{Conclusions}

It was found in this review of reported RCTs that acupuncture is an effective treatment for shoulder pain after stroke. Acupuncture can be considered as an adjuvant therapy in combination with exercise for rehabilitation of the stroke patients who are suffering from shoulder pain. Further trials concerning this topic should be conducted according to the highest methodological standards for certainty.

\section{Acknowledgments}

This research was supported by a grant (08-B-02) from Korea National Rehabilitation Research Institute.

\section{Disclosure Statement}

No competing financial interests exist.

\section{References}

1. Griffin JW. Hemiplegic shoulder pain. Phys Ther 1986;66: 1884-1893.

2. Turner-Stokes L, Jackson D. Shoulder pain after stroke: A review of the evidence base to inform the development of an integrated care pathway. Clin Rehabil 2002;16:276-298.

3. Snels IA, Dekker JH, van der Lee JH, et al. Treating patients with hemiplegic shoulder pain. Am J Phys Med Rehabil 2002;81:150-160.

4. Bohannon RW, Larkin PA, Smith MB, Horton MG. Shoulder pain in hemiplegia: Statistical relationship with five variables. Arch Phys Med Rehabil 1986;67:514-516.

5. Ernst E. Acupuncture: A critical analysis. J Intern Med 2006; 259:125-137.
6. Ernst E. Acupuncture. Lancet Oncol 2010;11:20.

7. Wu HM, Tang JL, Lin XP, et al. Acupuncture for stroke rehabilitation. Cochrane Database Syst Rev 2006;3:CD004131.

8. Zhang SH, Liu M, Asplund K, Li L. Acupuncture for acute stroke. Cochrane Database Syst Rev 2005;2:CD003317.

9. Sze FK, Wong E, Or KK, et al. Does acupuncture improve motor recovery after stroke? A meta-analysis of randomized controlled trials. Stroke 2002;33:2604-2619.

10. White AR, Ernst E. A systematic review of randomized controlled trials of acupuncture for neck pain. Rheumatology (Oxford) 1999;38:143-147.

11. Jadad AR, Moore RA, Carroll D, et al. Assessing the quality of reports of randomized clinical trials: Is blinding necessary? Control Clin Trials 1996;17:1-12.

12. van Tulder M, Furlan A, Bombardier C, Bouter L. Updated method guidelines for systematic reviews in the Cochrane Collaboration Back Review Group. Spine (Phila Pa 1976) 2003;28:1290-1299.

13. Shang YJ, Ma CC, Cha YY, et al. Clinical study on acupuncture combined with rehabilitation therapy for treatment of poststroke shoulder-hand syndrome. Chin Acupuncture Moxibustion 2008;28:331-333.

14. Zhu XJ, Gao WB, Yang XY. Shoulder subluxation after stroke treated with acupuncture combined with rehabilitative exercises. J Beijing Univ Chin Med 2007;14:23-25.

15. Zhao XF, Song H. Shoulder-hand syndrome after stroke treated with acupuncture of 30 cases. Chin J Information Trad Chin Med 2004;11:532-533.

16. Zhou GH. Observation of curative effect of acupuncture combined with rehabilitation training on hemiplegia patients with shoulder pain. Chin J Rehabil Theory Pract 2002; 8:244-245.

17. Xiong XR, Lin Q, Yao ZF, Yang CX. Wrist-ankle needle combined with rehabilitative exercises treated hemiplegia patients with shoulder pain of 36 cases. Fujian J TCM 2001; 32:33.

18. Zhang GP. The Rehabilitation Effects of Wrist-Ankle Needle on Omalgia for Post-stroke Patients. Fujian: Fujian University of Traditional Chinese Medicine, 2001.

19. Zhang HY. Study Comparatively the Curing Effect of Low Cycle Treatment and Electro-acupuncture Treatment in Curing the Shoulder Joint Half Dislocation Caused by Apoplexy Hemiplegy. Heilongjiang: Heilongjiang University of Chinese Medicine, 2008.

20. Park J, Hopwood V, White AR, Ernst E. Effectiveness of acupuncture for stroke: A systematic review. J Neurol 2001; 248:558-563.

21. Kong JC, Lee MS, Shin BC, et al. Acupuncture for functional recovery after stroke: A systematic review of sham-controlled randomized clinical trials. CMAJ 2010;182:1723-1729.

22. Perlow BW. Acupuncture: Its theory and use in general practice. Proc R Soc Med 1973;66:426-428.

23. Kaptchuk TJ. Acupuncture: theory, efficacy, and practice. Ann Intern Med 2002;136:374-383.

24. Lewith GT. How effective is acupuncture in the management of pain? J R Coll Gen Pract 1984;34:275-278.

Address correspondence to: Si-Woon Park, MD, MSCR Myongji Choonhey Rehabilitation Hospital Seoul Republic of Korea

E-mail: seanpark05@yahoo.co.kr 